\title{
Transtornos psiquiátricos menores em familiares cuidadores de usuários de Centros de Atenção Psicossocial: prevalência e fatores associados
}

\author{
Minor psychiatric disorders among family caregivers of users \\ of Psychosocial Care Centers: prevalence and associated factors
}

\author{
Carlos Alberto dos Santos Treichel (https://orcid.org/0000-0002-0440-9108) ${ }^{1}$ \\ Vanda Maria da Rosa Jardim (https://orcid.org/0000-0001-8320-4321) ${ }^{2}$ \\ Elaine Tomasi (https://orcid.org/0000-0001-7328-6044) ${ }^{2}$ \\ Luciane Prado Kantorski (https://orcid.org/0000-0001-9726-3162) ${ }^{2}$ \\ Michele Mandagará de Oliveira (https://orcid.org/0000-0002-7914-9339) ${ }^{2}$ \\ Valéria Cristina Christello Coimbra (https://orcid.org/0000-0001-5327-0141) ${ }^{2}$
}

${ }^{1}$ Universidade Estadual de Campinas. R. Tessália Vieira de Camargo 126, Cidade Universitária. 13083-887 Campinas SP Brasil.

carlos-treichel@

hotmail.com

${ }^{2}$ Universidade Federal de

Pelotas. Pelotas RS Brasil.

\begin{abstract}
This study aimed to identify the prevalence of the manifestation of Minor Psychiatric Disorders and its associated factors among $537 \mathrm{fa}$ mily caregivers of people living with psychological distress attended at 16 Psychosocial Care Centers (CAPS) located in the 21st health region of the state of Rio Grande do Sul. Considering a hierarchical theoretical model, the analysis adopted a Poisson regression to calculate the adjusted prevalence ratios. The prevalence of Minor Psychiatric Disorders found in the studied population was 42.1\% ( $N=226$ CI: 38\%-46,3\%). Factors associated with these disorders were being female ( $P R$ : 1.54), having a close relationship with the user especially fathers/mothers (PR: 2.00), low schooling, within a prevalence ratio of $P R: 1.85$ in the lowest stratum, presence of health problems (PR: 1.24); reporting neurological problems (PR: 3.02), low performance in the evaluation of the quality of life in the physical (PR: 1.84) and environmental (PR: 1.95) aspects, dissatisfaction with family relationships (PR: 1.56), lack of family support (PR: 1.25) and feeling of burden, with a prevalence ratio of PR: 2.61 among individuals with higher level of burden.
\end{abstract}

Key words Minor Psychiatric Disorders, SRQ20, Relatives, Caregivers, Community mental health services
Resumo Este estudo buscou identificar a prevalência e os fatores associados à manifestação de Transtornos Psiquiátricos Menores entre 537 cuidadores familiares de pessoas em sofrimento psíquico atendidas em 16 Centros de Atenção Psicossocial (CAPS) localizados na $21^{a}$ região de saúde do estado do Rio Grande do Sul. Para análise, munindo-se de um modelo teórico hierarquizado, utilizou-se regressão de Poisson com o cálculo das razões de prevalência ajustadas. A prevalência de Transtornos Psiquiátricos Menores encontrada na população estudada foi de 42,1\% ( $N=226$ IC: $38 \%-46,3 \%)$. Os fatores associados a esses transtornos foram: sexo feminino (RP: 1,54); vínculo próximo com o usuário, em especial pais/mães (RP: 2,00); baixa escolaridade, apresentando uma razão de prevalência de $R P$ : 1,85 no estrato mais baixo; possuir problemas de saúde (RP: 1,24); referir problemas de nervos (RP: 3,02); baixo desempenho de avaliação da qualidade de vida nos âmbitos físico $(R P: 1,84)$ e de meio ambiente (RP:1,95); insatisfação com as relações familiares (RP: 1,56); falta de apoio familiar (RP: 1,25) e sentimento de sobrecarga, para qual foi encontrado uma razão de prevalência de RP: 2,61 entre os indivíduos com maior nível de sobrecarga.

Palavras-chave Transtornos Psiquiátricos Menores, SRQ20, Familiares, Cuidadores, Serviços comunitários de saúde mental 


\section{Introdução}

A partir da reorganização das práticas de cuidado promovida pelos movimentos de reforma psiquiátrica no país na década de 80 , os familiares dos usuários de serviços comunitários de saúde mental passaram a ser importantes aliados no cuidado e na socialização de indivíduos em sofrimento psíquico ${ }^{1}$.

Contudo, apesar das evidências quanto à contribuição dessa parceria para um melhor prognóstico entre os usuários, diversos estudos ${ }^{2,3}$ têm documentado repercussões negativas da rotina de cuidados na vida dos familiares.

Entre essas repercussões, ganham destaque aquelas de cunho emocional, já que o avanço das pesquisas nesse campo aponta que muitos familiares acabam por experimentar sentimentos de depressão e ansiedade ${ }^{2}$.

Quando não atendem todos os critérios de doença mental de acordo com a Classificação Internacional das Doenças (CID-10), esses quadros de ansiedade e depressão são classificados como Transtornos Psiquiátricos Menores (TPM). Um processo que se refere aos estados de saúde envolvendo sintomas psiquiátricos não psicóticos, e inclui sintomas como: insônia, fadiga, irritabilidade, depressão, ansiedade, esquecimento, dificuldade de concentração e problemas somáticos ${ }^{4}$.

Até então, estudos realizados no país acerca desse desfecho têm indicado alta prevalência de Transtornos Psiquiátricos Menores entre familiares cuidadores de indivíduos em sofrimento psíquico quando comparados a resultados de população geral ${ }^{5-7}$.

Nesse sentido, ao levar em conta que os familiares constituem uma unidade que também precisa ser atendida em suas dificuldades, torna-se imperativa a necessidade de identificar quais características influem na ocorrência desses transtornos, já que esse pode ser um passo importante para o estabelecimento de práticas que previnam ou interfiram nesse desfecho.

Alguns autores ${ }^{6,7}$ que realizaram estudos nesse sentido indicam que diversos fatores, como problemas de saúde, sobrecarga e falta de apoio têm se mostrado relevantes para o estudo do tema. Contudo, pontuam também a incompletude das variáveis analisadas e a necessidade de incluir, especialmente, informações acerca do cuidado prestado e acerca dos indivíduos que são assistidos por esses familiares.

Nesse sentido, este estudo buscou incluir em sua investigação, além das variáveis já estudadas previamente, informações acerca do tempo na atividade de cuidador, tempo diário dedicado às atividades de cuidado, diagnóstico do usuário assistido e grau de dependência do usuário.

Dessa forma, espera-se que este estudo possa contribuir para expansão do entendimento quanto a seguinte questão de pesquisa: Qual é a prevalência e quais são os fatores associados à manifestação de Transtornos Psiquiátricos Menores entre familiares cuidadores de indivíduos em sofrimento psíquico usuários de Centros de Atenção Psicossocial?

\section{Metodologia}

Trata-se de um estudo transversal, realizado com familiares de usuários de Centros de Atenção Psicossocial nos municípios de abrangência da $21^{\text {a }}$ região de saúde do estado do Rio Grande do Sul no ano de 2016.

A $21^{\text {a }}$ região de saúde do estado do Rio Grande do Sul é composta por 22 municípios, dos quais 11 deles abrigam 23 CAPS. Destes, 4 são destinados à atendimento específico de usuários de álcool e outras drogas (AD), 3 são destinados ao atendimento da população infanto-juvenil e 16 CAPS I e II que atendem pacientes adultos com diversas demandas em saúde mental.

Neste estudo, optou-se por incluir todos os CAPS I e II da região supracitada, excluindo-se os serviços $\mathrm{AD}$, já que segundo os coordenadores desses serviços, em uma parte considerável dos casos, os usuários de CAPS do tipo AD optam por não envolver seus familiares no tratamento. Essa informação vai ao encontro dos resultados de um estudo realizado com usuários de álcool no Paraná ${ }^{8}$, cujo somente $16,6 \%$ dos usuários com consumo abusivo aceitaram envolver seus familiares na pesquisa.

Para definir o número de participantes foi realizado cálculo amostral. Para prevalência, o cálculo de amostra considerou uma frequência estimada de $50 \%$ com margem de 5 pontos e alfa (a) de 5\%, resultando na necessidade de um $\mathrm{N}$ $=384$. Já para associação, utilizando um poder de amostra de $80 \%$ com nível de significância de $5 \%$, relação de não expostos/expostos de 1, Risco Relativo de 1,3 e considerando prevalência de $40 \%$ em não exposto, obteve-se um indicativo de amostra de $\mathrm{N}=536$. Logo, acresceu-se ao maior $\mathrm{N}$ indicado $(\mathrm{N}=536) 30 \%$ de indivíduos a fim de considerar perdas e controle de fator de confusão. Dessa forma, pretendeu-se aplicar o questionário a 697 familiares de usuários de Centros de Atenção Psicossocial. 
Considerando a disparidade no número de habitantes por município, a fim de tornar a amostra representativa da população de familiares cuidadores de usuários de CAPS da $21^{\text {a }}$ Região de Saúde do Rio Grande do Sul, para definir o número de familiares a serem investigados com cada CAPS, levou-se em conta a proporcionalidade de indivíduos assistidos pelos serviços.

A seleção dos entrevistados se deu por amostragem aleatória simples. A partir das listas de usuários dos serviços, após sorteio, os familiares foram identificados e contatados e no caso de atenderem aos critérios de inclusão, convidados a participar do estudo. As entrevistas foram realizadas nos serviços. Foram critérios de inclusão: ser maior de idade; estar envolvido nas atividades de cuidado do usuário do CAPS. Foi critério de exclusão: ser cuidador contratado formalmente. O percentual de perdas no universo dos familiares correspondeu a $22,5 \%$, relacionado a recusas e dificuldades em responder o questionário, sendo assim, a população final acessada por este estudo foi de 537 familiares que foram submetidos à aplicação de um formulário pré-estruturado.

A coleta foi realizada por 24 entrevistadores selecionados e treinados previamente. $\mathrm{O}$ controle de qualidade dos dados foi realizado na codificação dos instrumentos de coleta e na revisão realizada pelos supervisores ao receber os questionários. A entrada dos dados no banco ocorreu no pacote estatístico Stata 11 (Stata Corp., College Station, Estados Unidos). Inconsistências nos dados foram avaliadas e corrigidas quando necessário.

$\mathrm{O}$ desfecho deste estudo foi a presença de Transtorno Psiquiátrico Menor. Para obtenção do desfecho foi utilizada a escala Self-Reporting Questionnaire (SRQ20). Este instrumento foi proposto pela Organização Mundial de Saúde para a detecção de Transtornos Psiquiátricos Menores, desenvolvido por Harding et al. ${ }^{9}$ e validada para o Brasil por Mari e Williams ${ }^{10}$.

A escala é composta por vinte questões com respostas do tipo sim ou não e conforme Harding et al. ${ }^{9}$, o ponto de corte, número de questões positivas que determinam a presença de um Transtorno Psiquiátrico Menor, pode apresentar variação considerável, de 5/6 a 10/11, dependendo do contexto cultural em que é aplicado, o que inclui contextos local e temporal. Este estudo assumiu os pontos de corte da validação brasileira ${ }^{10}$, que encontrou sensibilidade e especificidade de $83 \%$ e $80 \%$, respectivamente, quando aplicado o ponto de corte 6 para homens e 8 para mulheres.

Além da escala, foram incluídos nesse estudo variáveis acerca de dados sociodemográficos, trabalho, condições de saúde, qualidade de vida, relações familiares, características do indivíduo cuidado, apoio, aspectos relacionados ao cuidado e sobrecarga.

Para alguns dos aspectos supracitados, utilizaram-se escalas que pudessem providenciar dados mais sólidos e consistentes para as análises desenvolvidas. Entre os aspectos para os quais se utilizaram escalas estão: Características do Indivíduo Cuidado; Qualidade de Vida e Sobrecarga.

Para avaliação da qualidade de vida, utilizouse a escala WHOQOL-bref, composta por 26 questões divididas em quatro domínios: físico, psicológico, relações sociais e meio ambiente ${ }^{11}$. Os resultados foram transformado em escores de 0 a 100 e conforme versão adaptada ${ }^{12}$ foram considerados valores entre 0 (zero) e 40 (quarenta) como 'região de insatisfação'; de 41 (quarenta e um) a 70 (setenta), como 'região de satisfação parcial'; e acima de 71 (setenta e um) como 'região de satisfação'.

Para medir o grau de dependência dos indivíduos assistidos pelos familiares utilizou-se a escala de avaliação das Atividades Instrumentais da Vida Diária (AIVD) de Lawton e Brody ${ }^{13}$. A Escala varia de 0 a 16 pontos, a fim de categorizar os resultados conforme versão adaptada ${ }^{14}$ foram utilizados os seguintes pontos de corte: 0 a 5 dependência grave ou total; de 6 a 11 dependência moderada e de 12 a 16 ligeira dependência ou independente.

Para avaliar e classificar a sobrecarga entre os familiares foi utilizado a Escala Zarit Burden Interview $(\mathrm{ZBI})^{15}$. A pontuação da escala varia de 0 a 80 pontos, para classificação do grau de sobrecarga optou-se por utilizar os pontos de cortes propostos por Hebert et al. ${ }^{16}$, a saber: sobrecarga intensa (escore entre 61 e 88), sobrecarga moderada (escores entre 41 e 60), sobrecarga leve (escores entre 21 e 40) e ausência de sobrecarga (escores inferiores a 21).

Dessa forma as variáveis independentes incluídas no estudo foram: sexo (masculino; feminino); idade (18 a 40 anos; 41 a 50 anos; 51 a 60 anos; 61 anos ou mais); estado civil (solteiro; com companheiro); vínculo com o usuário (outros; irmã/irmão; filho/filha; cônjuge; pai/mãe); escolaridade ( 9 anos de estudo ou mais; 5 a 8 anos de estudo; 0 a 4 anos de estudo); trabalho remunerado (possui; não possui); renda per capita (1 salário mínimo ou mais; 0,5 a 1 salário mínimo; até 0,5 salário mínimo); problemas de saúde (não possui; possui); problemas de nervos (não possui; possui e não usa psicofármacos; possui e usa psicofármacos); realização de ativi- 
dades físicas (realiza; não realiza); principal cuidador (não é o principal cuidador; é o principal cuidador); divisão do cuidado (compartilha o cuidado; não compartilha o cuidado); tempo na atividade do cuidado ( 0 a 1 ano; 1 a 5 anos; 6 a 10 anos; mais de 10 anos); tempo diário no cuidado (0 a 8 horas; mais de 8 horas); diagnóstico do usuário (ansiedade; depressão; bipolaridade; esquizofrenia; retardo mental; álcool/drogas); grau de dependência do usuário (independente; parcialmente dependente; dependente); qualidade de vida - domínio físico (satisfeito; parcialmente satisfeito; insatisfeito); qualidade de vida - domínio psicológico (satisfeito; parcialmente satisfeito; insatisfeito); qualidade de vida - domínio das relações (satisfeito; parcialmente satisfeito; insatisfeito); qualidade de vida - domínio de meio ambiente (satisfeito; parcialmente satisfeito; insatisfeito); satisfação com as relações familiares (satisfeito; insatisfeito); apoio do CAPS (recebe; não recebe); apoio familiar (recebe; não recebe); sentimento de sobrecarga (sem sobrecarga; sobrecarga leve a moderada; sobrecarga moderada a intensa; sobrecarga intensa).

As análises foram conduzidas com o pacote estatístico Stata 11 (Stata Corp., College Station, Estados Unidos). Foi verificada a distribuição proporcional e intervalos de $95 \%$ de confiança (IC 95\%) para as variáveis categóricas. Na análise bruta, a prevalência de Transtornos Psiquiátricos menores foi calculada para cada variável independente e o teste chi-quadrado para heterogeneidade foi utilizado para identificar diferenças estatisticamente significativas entre os grupos ( $\mathrm{p}$ $<0,05)$.

A análise ajustada objetivou controlar possíveis fatores de confusão e foi feita por meio de regressão de Poisson, com o cálculo das razões de prevalência ajustadas. Foi usado o teste de Wald para testar heterogeneidade.

Os modelos de regressão levaram em consideração um modelo teórico hierárquico, adaptado a partir do modelo proposto por Treichel et al. ${ }^{7}$, e incluiu as variáveis em seis níveis. No primeiro, como determinante distal, foram incluídas as variáveis: sexo; idade; estado civil; vínculo com o usuário; escolaridade; trabalho remunerado e renda per capita. No segundo nível as variáveis: problemas de saúde; problemas de nervos e realização de atividades físicas. No terceiro nível: principal cuidador; divisão do cuidado; tempo na atividade do cuidado; tempo diário no cuidado; diagnóstico do usuário e grau de dependência do usuário. No quarto nível: qualidade de vida - domínio físico; qualidade de vida - domínio psico- lógico; qualidade de vida - domínio das relações; qualidade de vida - domínio de meio ambiente e satisfação com as relações familiares. No quinto nível: apoio do CAPS e apoio familiar. No sexto nível: sentimento de sobrecarga.

A entrada das variáveis na análise ajustada se deram nível a nível, todas as variáveis foram mantidas no modelo e ajustadas para aquelas do mesmo nível e de níveis anteriores.

O estudo foi submetido e aprovado pelo Comitê de Ética da Faculdade de Enfermagem da Universidade Federal de Pelotas seguindo as Normas e Diretrizes Regulamentadoras da Pesquisa Envolvendo Seres Humanos (Resolução CNS 466/2012). Os princípios éticos foram assegurados através de: consentimento livre e esclarecido; garantia do direito de não participação na pesquisa e anonimato. Nos casos em que durante as entrevistas foram identificadas situações em que havia necessidade imediata de atendimento, como, por exemplo, risco de suicídio ou presença de sintomas psicóticos, os sujeitos foram encaminhados para atendimento com profissionais do serviço onde estava ocorrendo a entrevista.

\section{Resultados}

Foram entrevistados 537 familiares, sendo 63,3\% do sexo feminino. A média de idade foi de 51,1 anos ( $\mathrm{DP}=13,3)$, com variação de 18 a 92 anos. Entre os entrevistados, $38,5 \%$ referiu possuir até 4 anos de estudo, enquanto $35,2 \%$ referiram ter estudado entre 5 e 8 anos e 26,2\% 9 anos de estudo ou mais. Quanto à renda, 37,7\% referiu renda per capita de até 0,5 salário mínimo, 41,2\% referiu renda per capita de até 1 salário mínimo e $21 \%$ renda per capita superior a um salário mínimo. 59,7\% dos entrevistados possuíam companheiro e $31 \%$ possuíam trabalho remunerado. A distribuição da população estudada quanto aos dados sociodemográficos pode ser observada na Tabela 1.

De acordo com a categorização proposta neste estudo, o rastreio de Transtornos Psiquiátricos Menores entre os entrevistados foi de $42,1 \%$ (N $=226$ IC: $38 \%-46,3 \%)$. A Tabela 2 apresenta a proporção encontrada para o desfecho de acordo com as características estudadas, nela ainda é possível observar a razão de prevalência bruta e ajustada para cada estrato, bem como seus respectivos p-valores.

$\mathrm{Na}$ análise bruta, 16 das 24 variáveis incluídas no modelo apresentaram associação com o desfecho $(\mathrm{p}<0,05)$, sendo elas: sexo $(\mathrm{p}=0,0006)$; 
Tabela 1. Características sociodemográficas da população estudada.

\begin{tabular}{|c|c|c|}
\hline Variáveis & $\mathbf{N}$ & $\%$ \\
\hline \multicolumn{3}{|l|}{ Sexo } \\
\hline Masculino & 197 & $36,7 \%$ \\
\hline Feminino & 340 & $63,3 \%$ \\
\hline \multicolumn{3}{|l|}{ Idade } \\
\hline 18 a 40 anos & 136 & $25,3 \%$ \\
\hline 41 a 50 anos & 107 & $19,9 \%$ \\
\hline 51 a 60 anos & 123 & $22,9 \%$ \\
\hline 61 anos ou mais & 171 & $31,8 \%$ \\
\hline \multicolumn{3}{|l|}{ Estado Civil } \\
\hline Solteiro & 216 & $40,2 \%$ \\
\hline Com companheiro & 321 & $59,8 \%$ \\
\hline \multicolumn{3}{|l|}{ Vínculo com o usuário } \\
\hline Outros & 79 & $14,7 \%$ \\
\hline Irmã/irmão & 74 & $13,8 \%$ \\
\hline Filho/filha & 72 & $13,4 \%$ \\
\hline Cônjuge & 131 & $24,4 \%$ \\
\hline Pai/Mãe & 181 & $33,7 \%$ \\
\hline \multicolumn{3}{|l|}{ Escolaridade } \\
\hline 9 anos de estudo ou mais & 141 & $26,3 \%$ \\
\hline 5 a 8 anos de estudo & 189 & $35,2 \%$ \\
\hline 0 a 4 anos de estudo & 207 & $38,5 \%$ \\
\hline \multicolumn{3}{|l|}{ Trabalho Remunerado } \\
\hline Possui & 169 & $31,5 \%$ \\
\hline Não possui & 368 & $68,5 \%$ \\
\hline \multicolumn{3}{|l|}{ Renda Per capita } \\
\hline 1 salário mínimo ou mais & 108 & $21,0 \%$ \\
\hline 0,5 a 1 salário mínimo & 212 & $41,2 \%$ \\
\hline Até 0,5 salário mínimo & 194 & $37,7 \%$ \\
\hline
\end{tabular}

vínculo com o usuário ( $\mathrm{p}=0,0270)$; escolaridade $(\mathrm{p}=0,0038)$; renda per capita $(\mathrm{p}=0,0040)$; problemas de saúde ( $\mathrm{p}=0,0055)$; problemas de nervos ( $\mathrm{p}=<0,0001)$; realização de atividades físicas $(\mathrm{p}=0,0265)$; tempo diário no cuidado ( $\mathrm{p}$ $=0,0100)$; grau de dependência do usuário ( $\mathrm{p}=$ $0,0087)$; qualidade de vida - domínio físico ( $\mathrm{p}=$ $<0,0001)$; qualidade de vida - domínio psicológico ( $\mathrm{p}=<0,0001)$; qualidade de vida - domínio das relações ( $\mathrm{p}=<0,0001)$; qualidade de vida domínio de meio ambiente $(\mathrm{p}=<0,0001)$; satisfação com as relações familiares $(\mathrm{p}=<0,0001)$; apoio familiar ( $p=0,0042)$; sentimento de sobrecarga $(\mathrm{p}=<0,0001)$.

Já na análise ajustada, perderam força de associação as variáveis renda per capita ( $\mathrm{p}=$ $0,1632)$; realização de atividades físicas ( $\mathrm{p}=$ $0,368)$; principal cuidador $(\mathrm{p}=0,997)$; tempo diário no cuidado ( $\mathrm{p}=0,638)$; grau de dependência do usuário $(\mathrm{p}=0,336)$; qualidade de vida
- domínio psicológico $(\mathrm{p}=0,0970)$ e qualidade de vida - domínio das relações $(\mathrm{p}=0,6996)$.

Dessa forma, estiveram associadas com o desfecho as variáveis: sexo $(\mathrm{p}=0,001)$; vínculo com o usuário ( $\mathrm{p}=0,0197)$; escolaridade $(\mathrm{p}=$ $0,0013)$; problemas de saúde $(\mathrm{p}=0,049)$; problemas de nervos ( $\mathrm{p}=<0,0001)$; qualidade de vida - domínio físico ( $\mathrm{p}=0,0013$ ); qualidade de vida - domínio de meio ambiente $(\mathrm{p}=0,0051)$; satisfação com as relações familiares $(\mathrm{p}=<0,0001)$; apoio familiar $(\mathrm{p}=0,043)$ e sentimento de sobrecarga $(\mathrm{p}=<0,0001)$.

Indivíduos do sexo feminino apresentaram razão de prevalência 54\% maior no rastreio para Transtornos Psiquiátricos Menores.

Maiores razões de prevalência em apresentar o desfecho foram encontradas entre os estratos que indicavam vínculos familiares mais próximos, sendo pai/mãe e irmã/irmão aqueles que apresentaram maior razão de prevalência para rastreio do desfecho ( 2 vezes e 2,04 vezes maior respectivamente).

Indivíduos com menor escolaridade apresentaram maior razão de prevalência no rastreio para Transtornos Psiquiátricos Menores. Comparados aos sujeitos com 9 anos de estudo ou mais, aqueles com escolaridade entre 5 e 8 anos de estudo apresentaram razão de prevalência 59\% maior de manifestação do desfecho. No mesmo sentido, indivíduos sem escolaridade ou com até 4 anos de estudo apresentaram razão de prevalência $85 \%$ maior.

Possuir algum problema de saúde foi marcador de uma razão de prevalência $24 \%$ maior para manifestação de Transtornos Psiquiátricos Menores. Da mesma forma, indivíduos que relataram possuir problemas de nervos apresentaram razão de prevalência 2,28 vezes maior de apresentar o desfecho quando não faziam uso de psicofármacos e 3,02 vezes maior no caso de além do relato, estarem tomando algum psicofármaco.

Insatisfação com domínio físico da medida de qualidade de vida esteve associado com maior risco de manifestação de Transtornos Psiquiátricos Menores. Comparados a indivíduos satisfeitos com o domínio supracitado, sujeitos insatisfeitos apresentaram razão de prevalência $84 \%$ maior para rastreio do desfecho.

Da mesma forma, indivíduos insatisfeitos com o domínio de meio ambiente da medida de qualidade de vida apresentaram maior razão de prevalência para rastreio de Transtornos Psiquiátricos Menores. Comparados aos indivíduos satisfeitos, aqueles que estavam parcialmente satisfeitos apresentaram razão de prevalência 95\% 
Tabela 2. Prevalência e razão de prevalência para Transtornos Psiquiátricos Menores de acordo com variáveis independentes em familiares de usuários de CAPS.

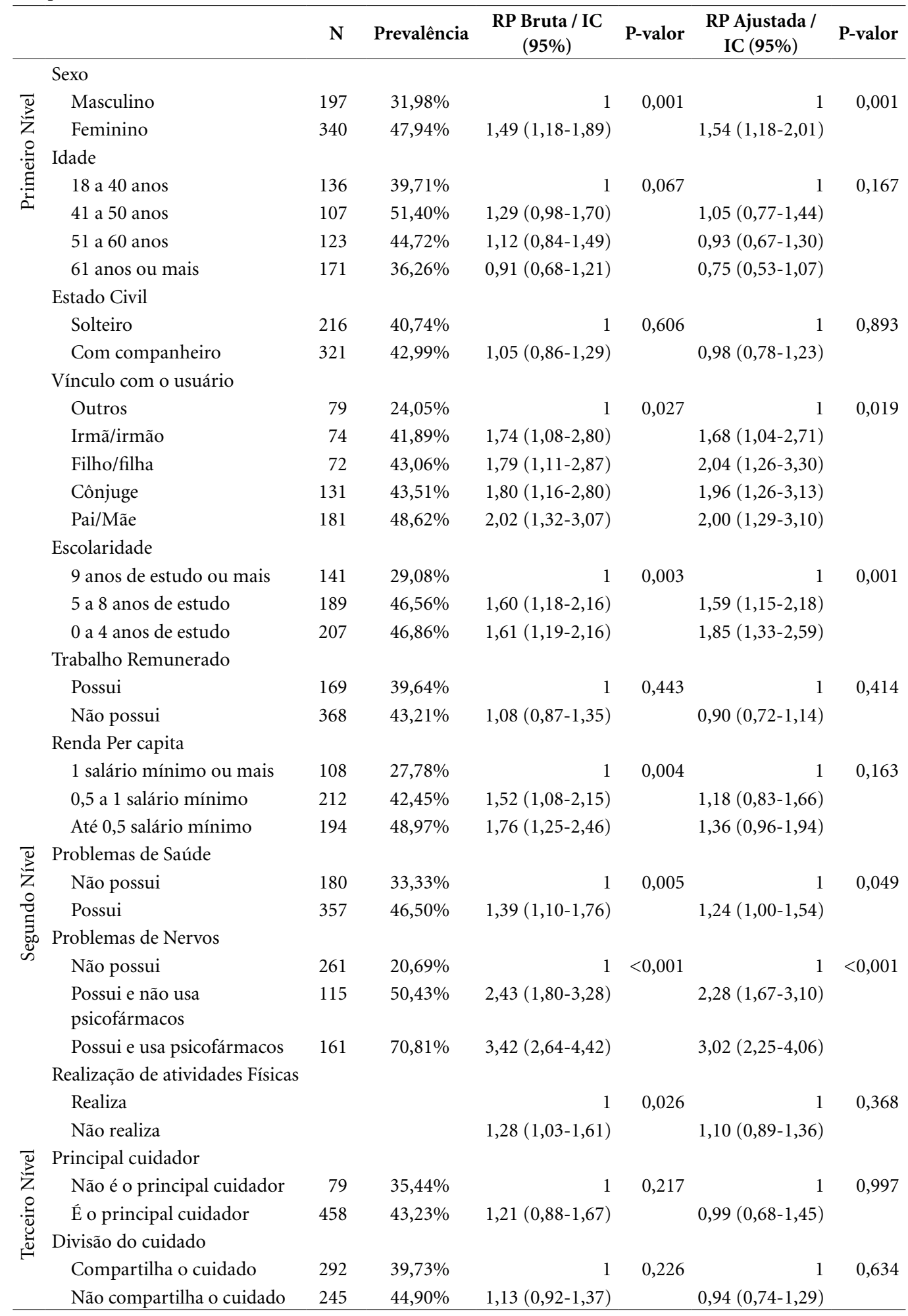


Tabela 2. Prevalência e razão de prevalência para Transtornos Psiquiátricos Menores de acordo com variáveis independentes em familiares de usuários de CAPS.

\begin{tabular}{|c|c|c|c|c|c|c|c|}
\hline & & $\mathbf{N}$ & Prevalência & $\begin{array}{c}\text { RP Bruta / IC } \\
(95 \%)\end{array}$ & P-valor & $\begin{array}{l}\text { RP Ajustada / } \\
\text { IC (95\%) }\end{array}$ & P-valor \\
\hline \multirow{19}{*}{ 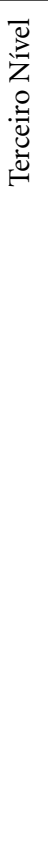 } & $\begin{array}{l}\text { Tempo na atividade do } \\
\text { cuidado }\end{array}$ & & & & & & \\
\hline & 0 a 1 ano & 63 & $50,79 \%$ & 1 & 0,289 & 1 & 0,172 \\
\hline & 1 a 5 anos & 173 & $39,31 \%$ & $0,77(0,56-1,05)$ & & $0,74(0,53-1,02)$ & \\
\hline & 6 a 10 anos & 122 & $38,52 \%$ & $0,75(0,54-1,05)$ & & $0,74(0,52-1,04)$ & \\
\hline & Mais de 10 anos & 171 & $44,44 \%$ & $0,87(0,65-1,17)$ & & $0,80(0,51-1,97)$ & \\
\hline & Tempo diário no cuidado & & & & & & \\
\hline & 0 a 8 horas & 186 & $33,87 \%$ & 1 & 0,010 & 1 & 0,638 \\
\hline & Mais de 8 horas & 290 & $46,21 \%$ & $1,36(1,07-1,72)$ & & $1,06(0,82-1,36)$ & \\
\hline & Diagnóstico do usuário & & & & & & \\
\hline & Ansiedade & 35 & $34,29 \%$ & 1 & 0,391 & 1 & 0,056 \\
\hline & Depressão & 176 & $38,64 \%$ & $1,12(0,68-1,84)$ & & $1,83(1,15-2,90)$ & \\
\hline & Bipolaridade & 69 & $46,38 \%$ & $1,35(0,80-2,28)$ & & $1,53(0,90-2,60)$ & \\
\hline & Esquizofrenia & 169 & $44,38 \%$ & $1,29(0,70-2,11)$ & & $1,59(1,00-2,54)$ & \\
\hline & Retardo mental & 38 & $52,63 \%$ & $1,53(0,88-2,65)$ & & $2,13(1,23-3,68)$ & \\
\hline & Álcool/Drogas & 21 & $52,38 \%$ & $1,52(0,82-2,82)$ & & $2,29(1,13-4,63)$ & \\
\hline & Grau de dependência do usuár & & & & & & \\
\hline & Independente & 90 & $54,44 \%$ & 1 & 0,008 & 1 & 0,420 \\
\hline & Parcialmente dependente & 187 & $43,32 \%$ & $0,79(0,61-1,02)$ & & $1,00(0,76-1,32)$ & \\
\hline & Dependente & 260 & $36,92 \%$ & $0,67(0,52-0,86)$ & & $0,85(0,63-1,16)$ & \\
\hline \multirow{19}{*}{ 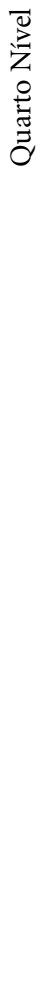 } & $\begin{array}{l}\text { Qualidade de vida (domínio } \\
\text { físico) }\end{array}$ & & & & & & \\
\hline & Satisfeito & 276 & $22,83 \%$ & 1 & $<0,001$ & 1 & 0,001 \\
\hline & Parcialmente satisfeito & 227 & $58,59 \%$ & $2,56(2,01-3,27)$ & & $1,68(1,25-2,24)$ & \\
\hline & Insatisfeito & 34 & $88,24 \%$ & $3,86(3,01-4,96)$ & & $1,84(1,28-2,65)$ & \\
\hline & $\begin{array}{l}\text { Qualidade de vida (domínio } \\
\text { psicológico) }\end{array}$ & & & & & & \\
\hline & Satisfeito & 297 & $24,24 \%$ & 1 & $<0,001$ & 1 & 0,097 \\
\hline & Parcialmente satisfeito & 210 & $59,05 \%$ & $2,43(1,93-3,06)$ & & $1,23(0,96-1,58)$ & \\
\hline & Insatisfeito & 30 & $100 \%$ & $4,12(3,37-5,04)$ & & $1,39(1,02-1,91)$ & \\
\hline & $\begin{array}{l}\text { Qualidade de vida (domínio } \\
\text { das relações) }\end{array}$ & & & & & & \\
\hline & Satisfeito & 291 & $29,55 \%$ & 1 & $<0,001$ & 1 & 0,699 \\
\hline & Parcialmente satisfeito & 210 & $52,38 \%$ & $1,77(1,42-2,20)$ & & $0,91(0,72-1,13)$ & \\
\hline & Insatisfeito & 36 & $83,33 \%$ & $2,81(2,24-3,54)$ & & $0,91(0,68-1,22)$ & \\
\hline & $\begin{array}{l}\text { Qualidade de vida (domínio } \\
\text { meio ambiente) }\end{array}$ & & & & & & \\
\hline & Satisfeito & 150 & $14,67 \%$ & 1 & $<0,001$ & 1 & 0,005 \\
\hline & Parcialmente satisfeito & 355 & $50,14 \%$ & $3,41(2,29-5,10)$ & & $2,02(1,32-3,09)$ & \\
\hline & Insatisfeito & 32 & $81,25 \%$ & $5,53(3,63-8,43)$ & & $1,95(1,14-3,34)$ & \\
\hline & $\begin{array}{l}\text { Satisfação com as relações } \\
\text { familiares }\end{array}$ & & & & & & \\
\hline & Satisfeito & 418 & $32,54 \%$ & 1 & $<0,001$ & 1 & $<0,001$ \\
\hline & Insatisfeito & 119 & $75,63 \%$ & $2,32(1,95-2,76)$ & & $1,56(1,27-1,93)$ & \\
\hline \multirow{6}{*}{ 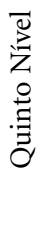 } & Apoio do CAPS & & & & & & \\
\hline & Recebe & 346 & $41,62 \%$ & 1 & 0,767 & 1 & 0,187 \\
\hline & Não recebe & 191 & $42,93 \%$ & $1,03(0,83-1,23)$ & & $0,86(0,69-1,07)$ & \\
\hline & Apoio familiar & & & & & & \\
\hline & Recebe & 295 & $37,29 \%$ & 1 & 0,004 & 1 & 0,043 \\
\hline & Não recebe & 242 & $47,93 \%$ & $1,28(1,05-1,56)$ & & $1,25(1,00-1,55)$ & \\
\hline
\end{tabular}




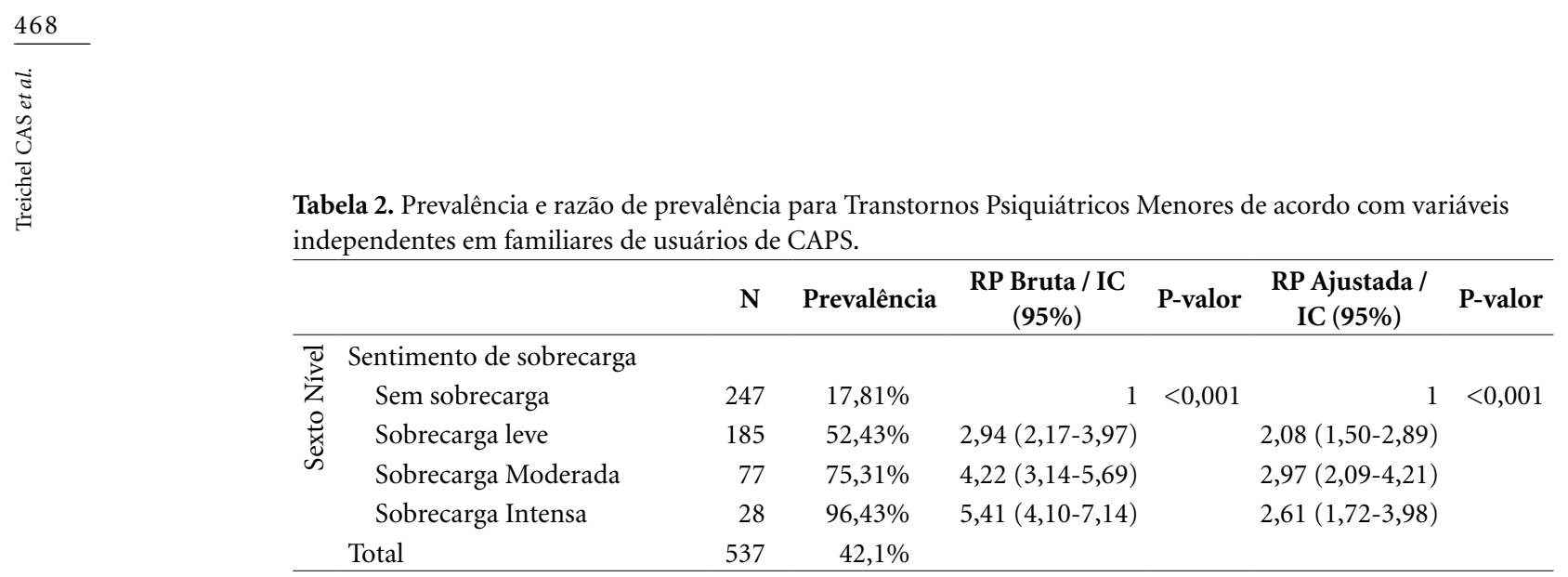

maior do desfecho e entre os insatisfeitos a razão de prevalência foi 2,02 vezes maior.

Quanto às relações familiares, indivíduos que se mostraram insatisfeitos com sua relação familiar apresentaram razão de prevalência 56\% maior para rastreio positivo para Transtornos Psiquiátricos Menores. Ainda no âmbito familiar, indivíduos que referiram não receber apoio de sua família para cuidar do usuário apresentaram razão de prevalência $25 \%$ maior para manifestação do desfecho.

Maior razão de prevalência para o rastreio positivo para Transtornos Psiquiátricos Menores foram encontradas quanto maior o grau de sobrecarga apresentado pelos sujeitos investigados. Comparados aos indivíduos não sobrecarregados, aqueles que apresentavam sobrecarga leve apresentaram uma razão de prevalência 2,08 vezes maior para apresentação do desfecho. No mesmo sentido, indivíduos com sobrecarga moderada apresentaram razão de prevalência 2,61 vezes maior e indivíduos com sobrecarga intensa apresentaram razão de prevalência 2,97 vezes maior.

\section{Discussão}

A prevalência de Transtornos Psiquiátricos Menores na população estudada foi de $42,1 \%(\mathrm{~N}=$ 226 IC: $38 \%-46,3 \%)$. Esta prevalência é próxima àquelas encontradas em outros estudos conduzidos com populações semelhantes. Em populações de familiares cuidadores de usuários de Centros de Atenção Psicossocial, estudos como os de Tomasi et al. ${ }^{5}$, Quadros et al. ${ }^{6}$ e Treichel et al. ${ }^{7}$ encontraram prevalências de $41 \%, 49 \%$ e $46,9 \%$ respectivamente. Nesse sentido, considerando estudos de base populacional conduzidos anteriormente no país ${ }^{17,18}$, este estudo corrobora com a perspectiva de que os familiares cuidado- res constituem uma população de risco para manifestação de Transtornos Psiquiátricos Menores.

$\mathrm{Na}$ análise ajustada, ser do sexo feminino esteve associado ao desfecho. Este resultado corrobora com achados prévios de Treichel et al. ${ }^{7}$, que também encontrou maior risco de manifestação de Transtornos Psiquiátricos Menores em mulheres cuidadoras em saúde mental do que em homens na mesma situação.

Além da variável sexo, as variáveis sociodemográficas que estiveram associadas ao desfecho foram vínculo com o usuário e escolaridade. Estes achados corroboram com achados prévios de Treichel et al. ${ }^{7}$ no sentido de que vínculos mais próximos e menor escolaridade são fatores que contribuem para maiores chances de manifestação de Transtornos Psiquiátricos Menores. Menor escolaridade esteve associada com o desfecho ainda no estudo de Quadros et al. ${ }^{6}$.

Assim como nos estudos de Quadros et al. ${ }^{6} \mathrm{e}$ Treichel et al. ${ }^{7}$, embora renda estivesse associada com o desfecho na análise bruta, ao ser inserida no modelo, essa variável perdeu força de associação.

Possuir problemas de saúde tem sido documentado como um dos fatores associados à positividade no SRQ-20,7.7. Dessa forma, podese apontar que os resultados corroboram com a literatura nesse sentido. Ressalta-se ainda que insatisfação com o domínio físico da escala de medida para qualidade de vida esteve fortemente associada a manifestação de Transtornos Psiquiátricos Menores entre a população estudada. Contudo é importante ressaltar que embora a relação entre problemas de saúde e Transtornos Psiquiátricos Menores venha sendo sistematicamente documentada, especialmente em familiares cuidadores, a direção dessa relação não é clara.

Enquanto no estudo de Tomasi et al. ${ }^{5}$ uma parcela de $26 \%$ dos familiares referiram possuir problemas de nervos, neste estudo $51 \%$ dos entrevistados referiram esses problemas. Na análi- 
se ajustada estas situações estiveram fortemente associadas à manifestação de Transtornos Psiquiátricos Menores, especialmente nos casos em que além de relatar possuir um problema de nervos, os entrevistados realizavam uso de algum psicofármaco. Ressalta-se que 30\% dos familiares acessados pelo estudo faziam uso de algum desses medicamentos. Dessa forma, é necessário atentar para dois aspectos importantes: a existência de uma parcela importante de familiares que já está adoecida e em tratamento, e a possibilidade de interferência da ação dos psicofármacos em sintomas utilizados para rastreio dos Transtornos Psiquiátricos Menores na escala utilizada neste estudo.

Uma das principais demandas de estudos realizados anteriormente quanto ao tema era a necessidade de informações acerca do cuidado prestado e principalmente acerca do indivíduo cuidado pelos familiares estudados ${ }^{6,7}$. Nesse sentido, este estudo conta como ponto forte o agrupamento de uma série de variáveis que contribuem para expansão das investigações quanto a esses aspectos.

Contudo, embora variáveis como tempo diário no cuidado e grau de dependência do usuário tivessem apresentado força de associação com o desfecho na análise bruta, após ajuste, essas variáveis não estiveram associadas à manifestação de Transtornos Psiquiátricos Menores entre os familiares estudados. $\mathrm{Na}$ análise bruta, indivíduos que prestavam mais de 8 horas diárias de cuidado apresentavam razão de prevalência $36 \%$ maior na apresentação do desfecho, contudo, na análise ajustada essa variável perdeu força de associação. Esse fato pode estar relacionado com a divisão da carga do cuidado em aspectos objetivos e subjetivos. Um maior tempo dispensado ao cuidado indica uma maior carga objetiva, contudo, pode não traduzir os elementos subjetivos, apontados previamente na literatura como importantes marcadores de sobrecarga e adoecimento emocional $^{15}$.

Quanto ao grau de dependência do usuário cuidado, cabe ressaltar que embora na análise bruta essa variável tivesse demonstrado associação com o desfecho, essa associação ocorreu num sentido inverso ao esperado. Embora com base em estudos prévios ${ }^{5}$ que apontaram maior sobrecarga e risco de adoecimento emocional de acordo com maior grau de dependência do indivíduo cuidado, neste estudo, na análise bruta, quanto maior o grau de dependência do usuário, menor foi o risco de manifestação de transtornos psiquiátricos nos familiares.
Em seu estudo Treichel et al. ${ }^{7}$ indica uma forte associação da insatisfação com a qualidade de vida e a manifestação de Transtornos Psiquiátricos Menores. Nesse sentido, por tomar a qualidade de vida como um entendimento complexo que leva em conta uma série de valores relacionados a diferentes aspectos, este estudo buscou dividir a satisfação com a qualidade de vida entre os quatro domínios propostos pela organização mundial da saúde para estudo da qualidade de vida por meio do instrumento WHOQOL $-\mathrm{BREF}^{19}$. Na análise bruta todos os domínios da escala WHOQOL-BREF apresentaram força de associação com o desfecho, no sentido de quanto menor a satisfação com o domínio, maior o risco de manifestação de Transtornos Psiquiátricos Menores. Contudo, na análise ajustada, apenas os domínios relacionados à saúde física e meio ambiente estiveram associados ao desfecho.

Insatisfação com as relações familiares foi um importante marcador da presença de Transtornos Psiquiátricos Menores neste estudo. Ressaltase que estudos prévios $^{20}$ já vêm documentando piores desfechos na qualidade de vida entre cuidadores oriundos de família com pior funcionamento familiar. Dessa forma, pode-se destacar que este achado chama atenção para uma interface importante do cuidado dentro do contexto familiar, a funcionalidade da família pode ser afetada pelas relações de cuidado. Nesse sentido, os resultados deste estudo tencionam a necessidade de que as perspectivas de funcionamento familiar sejam levadas em conta na abordagem dos serviços tanto para o acompanhamento dos indivíduos assistidos, quanto para o monitoramento das repercussões do cuidado na vida dessas pessoas e do estabelecimento do plano de cuidados do indivíduo em sofrimento psíquico e dos familiares envolvidos no plano terapêutico.

Ainda quanto às relações familiares, falta de apoio familiar esteve associada à manifestação de Transtornos Psiquiátricos Menores. Resultado que corrobora com a perspectiva apontada por Quadros et al. ${ }^{6}$ de que apoio social é fator de proteção quanto ao desfecho. Em seu estudo, os autores citam que o sentimento de apoio por parte de entes queridos pode melhorar a adaptação para as circunstâncias particulares de estresse e diminuir o efeito dos eventos produtores desse estresse, de forma que mesmo que não sejam evitados, os mesmos podem passar a ter menores consequências.

Sentimento de sobrecarga esteve fortemente associada ao rastreio positivo para Transtornos Psiquiátricos Menores, tanto na análise bruta 
quanto ajustada, o p valor encontrado para essa associação foi $p=<0,0001$. Ressalta-se que quanto maior o grau de sobrecarga observado, maior foi o risco da manifestação desses transtornos. É possível apontar que a variável sobrecarga vem sendo repetidamente citada por trabalhos dentro do campo da saúde mental como um disparador das repercussões negativas na vida dos cuidadores familiares ${ }^{21}$, contudo, embora recorrente nesse contexto, não se trata de aspecto fácil de ser abordado. Alguns autores citam, por exemplo, que para alguns cuidadores, a sobrecarga é algo inerente às atividades do cuidado e está atrelada a sua responsabilidade de cuidar de "parentes doentes"22. Dessa forma, evidencia-se que, em geral, entre as expectativas desses cuidadores, não está a ausência de sobrecarga, mas necessidade de apoio e suporte.

A expansão desse entendimento pode ajudar na interpretação dos resultados deste estudo. Embora se deva considerar a importância de cada variável individualmente, a análise multivariada parece sugerir que os aspectos que efetivamente têm exercido influência sobre o desfecho na amostra estudada estão relacionados às condições situacionais e de apoio e organização familiar.

Do ponto de vista das condições situacionais, aspectos como a presença de uma injúria física e pior desempenho na qualidade de vida nos domínios físico e de meio ambiente indicam um ambiente menos favorável para as adaptações necessárias a partir do adoecimento seja mental ou físico de um membro da família. Já quanto ao apoio e organização familiar, ressalta-se que a necessidade de reorganização da estrutura e da rotina familiar é um dos pontos frequentemente citados na literatura como um dos grandes desafios a serem enfrentados pelos grupos familiares a partir do convívio com o sofrimento psíquico de um de seus membros ${ }^{23,24}$. Autores como Santin e $\mathrm{Klafke}^{24}$ citam que a depender da posição que o membro adoecido ocupa na família, o momento de definição dos cuidadores pode tanto unir como agravar tensões entre os familiares.

Dessa forma, cabe reflexão quanto ao papel dos serviços de saúde mental frente a essas situações. Ressalta-se que dentro do processo de cuidado, em geral, apenas um dos familiares é inserido no projeto terapêutico do paciente e constitui-se como referência para as ações de cuidado que ultrapassam o espaço assistido pelo serviço ${ }^{23}$. Esse fator pode contribuir para perpetuação de práticas pouco saudáveis para as relações intrafamiliares, como a transferência de responsabi- lidades para um único indivíduo, favorecendo o sentimento de sobrecarga ${ }^{25}$. Uma alternativa em favor da mudança desse paradigma talvez seja o estabelecimento de práticas que envolvam e valorizem todos os membros da família do indivíduo em sofrimento psíquico. Iniciativas nesse sentido vêm ocorrendo desde os anos 80 em outras partes do mundo, como por exemplo, na Finlândia, através do "Diálogo Aberto" e convergem em melhores prognósticos entre os indivíduos em sofrimento psíquico e suas famílias ${ }^{25-27}$.

$\mathrm{Na}$ perspectiva da abordagem do "Diálogo aberto" toda rede social do indivíduo em sofrimento psíquico, ou seja, sua família, seus amigos e vínculos significativos, é incluída no tratamento. Esses sujeitos estão presentes em todas as reuniões, sem que nenhuma decisão seja tomada fora delas. Estudos acerca dos prognósticos desse modelo têm apontado uma relação direta entre os resultados exitosos do método com a perspectiva de que essa prática assegura a continuidade psicológica em todas as etapas do tratamento com foco no diálogo entre as pessoas implicadas na situação, valorizando a construção conjunta de entendimentos e saídas ${ }^{25-27}$. Nesse sentido, sugere-se que para os avanços da pesquisa na área, sejam realizados estudos que investiguem o impacto da implementação de ações terapêuticas que incluam todo grupo familiar e afetivo dos indivíduos em sofrimento psíquico na manifestação de Transtornos Psiquiátricos Menores.

Cabe destacar que este estudo possui um delineamento de corte transversal, tendo exposição e desfecho avaliados no mesmo momento. Dessa forma, sua leitura deve ser realizada considerando a causalidade reversa como uma limitação. Contudo, os resultados da análise ajustada sugerem que as associações encontradas entre as variáveis e o desfecho não se devem ao acaso, mesmo que não seja possível conhecer o sentido das mesmas.

\section{Conclusão}

A prevalência de Transtornos Psiquiátricos Menores encontrada na população estudada foi próxima àquelas encontradas anteriormente em cuidadores familiares de pessoas em sofrimento psíquico no país. Esse achado reforça a ideia de que esta é uma população de risco para o adoecimento emocional e psíquico e aponta a necessidade de acompanhamento da mesma através de ações de rastreamento, prevenção e intervenção dessas situações. 
Os fatores associados aos Transtornos Psiquiátricos Menores na população estudada foram: sexo feminino; vínculo próximo com o usuário, em especial pais/mães; baixa escolaridade; possuir problemas de saúde; referir problemas de nervos; baixo desempenho de avaliação da qualidade de vida nos âmbitos físico e de meio ambiente; insatisfação com as relações familiares; falta de apoio familiar e sentimento de sobrecarga.
Apesar de estudos prévios indicarem a necessidade de incluir variáveis acerca das características do cuidado e acerca dos indivíduos assistidos pelos cuidadores, após ajuste, este estudo não encontrou associação da manifestação de Transtornos Psiquiátricos Menores com essas variáveis. Por outro lado, os resultados deste estudo apontaram que entre os fatores que efetivamente impactam no desfecho, destacam-se o apoio e organização familiar.

\section{Colaboradores}

CAS Treichel trabalhou na concepção e delineamento da pesquisa, análise e interpretação dos dados, e redação do manuscrito. VMR Jardim, E Tomasi, e LP Kantorski trabalharam na concepção e delineamento da pesquisa e na análise e interpretação dos dados. MM Oliveira e VCC Coimbra trabalharam na redação e revisão crítica do manuscrito. 


\section{Referências}

1. Brasil. Ministério da Saúde (MS). Secretaria de Atenção à Saúde. DAPES. Coordenação Geral de Saúde Mental, Álcool e Outras Drogas. Saúde Mental no SUS: Cuidado em Liberdade, Defesa de Direitos e Rede de Atenção Psicossocial. Relatório de Gestão 2011-2015. Brasília: MS; 2016.

2. Bandeira M, Calzavara MCP, Castro I. Estudo de validade da escala de sobrecarga de familiares cuidadores de pacientes psiquiátricos. J Bras Psiquiatr 2008; 57(2):98-104.

3. Bielemann VLM, Kantorski LP, Borges LR, Chiavagatti FG, Willrich JQ, Souza AF, Heck RM. A inserção da família nos centros de atenção psicossocial sob a ótica de seus atores sociais. Texto Contexto Enferm 2009; 18(1):131-139.

4. Tavares JP, Beck CLC, Magnago TSBS, Greco PBT, Prestes FC, Silva RM. Produção científica sobre os distúrbios psíquicos menores a partir do self report questionnarie. R Enferm UFSM 2011; 1(1):113-123.

5. Tomasi E, Rodrigues JO, Feijó GP, Facchini LA, Piccini PX, Thumé E, Silva RA, Gonçalves H. Sobrecarga em familiares de portadores de sofrimento psíquico que frequentam Centros de Atenção Psicossocial. Saúde Debate 2010; 34(84):159-167.

6. Quadros LC, Gigante DP, Kantorski LP, Jardim VMR. Minor psychiatric disorders in family caregivers of users of Psychosocial Care Centers in southern Brazil. Cad Saude Publica 2012; 28(1):95-103.

7. Treichel CAS, Jardim VMR, Kantorski LP, Oliveira MM, Coimbra VCC, Neutzling AS. Minor psychiatric disorders and their associations in family caregivers of people with mental disorders. Cien Saude Colet 2017; 22(11):3567-3577.

8. Lopes APT, Ganassin GS, Marcon SS, Decesaro MN. Abuso de bebida alcoólica e sua relação no contexto familiar. Estud Psicol 2015; 20(1):22-30.

9. Harding TW, Arango MV, Baltazar J, Climent CE, Ibrahim HH, Ladrido-Ignacio L, Murthy RS, Wig NN. Mental disorders in primary health care: A study of the frequency and diagnosis in four developing countries. Psychol Med 1980; 10(2):231-241.

10. Mari JJ, Williams P. A validity study of a psychiatric screening questionnaire (SRQ-20) in primary care in the city of Sao Paulo. Br J Psychiatry 1986; 148:23-26.

11. Fleck MPA, Louzada S, Xavier M, Chachamovich E, Vieira G, Santos L, Pinzon V. Aplicação da versão em português do instrumento abreviado de avaliação da qualidade de vida "WHOQOL-bref”. Rev Saude Publica 2000; 34(2):178-183.

12. Saupe R, Nietche EA, Cestari ME, Giorgi MDM, Krahl M. Qualidade de vida dos acadêmicos de enfermagem. Rev Latino-Am Enfermagem 2004; 12(4):636-642.

13. Lawton MP, Brody EM. Assesment of older people: self-maintaining and instrumental activities of daily living. Gerontologist 1969; 9(3):179-185.

14. Azeredo Z, Matos E. Grau de dependência em doentes que sofreram AVC. Rev Fac Med Lisboa 2003; 8(4):199-204.

15. Scazufca M. Brazilian version of the Burden Interview scale for the assessment of burden of care in careers of people with mental illness. Rev Bras Psiquiatr 2002; 24(1):12-17.
16. Hérbert R, Bravo G, Préville M. Reliability, validity, and reference values of the Zarit Burden Interview for assessing informal caregivers of community-dwelling older persons with dementia. Canadian J Aging 2000; 19(4):494-507.

17. Maragno L, Goldbaum M, Gianini RJ, Novaes HMD, Cesar CLG. Prevalência de Transtorno mental comum em populações atendidas pelo Programa Saúda da Família (QUALIS) no Município de São Paulo, Brasil. Cad Saude Publica 2006; 22(8):1639-1648.

18. Gonçalves DM, Kapczinski F. Transtornos mentais em comunidade atendida pelo Programa Saúde da Família. Cad Saude Publica 2008: 24(7):1641-1650.

19. WHOQOL Group. The World Health Organization quality of life assessment (WHOQOL): position paper from the World Health Organization. Soc Sci Med 1995; 41(10):1403-1410.

20. Rodríguez-Sánchez E, Pérez-Peñaran A, Losada-Bal$\operatorname{tar}$ A, Pérez-Arechaederra D, Gómez-Marcos MA, Patino-Alonso MC, García-Ortiz L. Relationships between quality of life and family function in caregiver. BMC Fam Pract 2011; 12:19.

21. Treichel CAS, Jardim VMR, Kantorski LP, Vasem ML, Neutzling AS. Clustering of minor psychiatric disorders and burden among family caregivers of individuals with mental illness. Cien Saude Colet 2016; 21(2):585-590.

22. Wong DF, Lam AY, Chan SK, Chan SF. Quality of life of caregivers with relatives suffering from mental illness in Hong Kong: roles of caregiver characteristics, caregiving burdens, and satisfaction with psychiatric services. Health Qual Life Outcomes 2012; 10:15.

23. Oliveira RMP, Loyola CM. Família do paciente psiquiátrico: o retrato de uma ilustre desconhecida. Acta Sci Health Sci 2001; 26(1):213-222.

24. Santin G, Klafke TE. A família e o cuidado em saúde mental. Barbaroi 2011; 34:146-160.

25. Centeno RM, Fadda SMN, Jiménez EV, Castañeda RC. Complexidade e esquizofrenia. Rev Bras Psicoter 2013; 15(1):59-71.

26. Seikkula J, Alakare B, Aaltonen J. The Comprehensive Open-Dialogue Approach in Western Lapland: II. Long-term stability of acute psychosis outcomes in advanced community care. Psychosis 2011; 3(3):192204.

27. Kłapciński MM, Rymaszewska J. Open Dialogue Approach - about the phenomenon of Scandinavian Psychiatry. Psychiatr Pol 2013; 49(6):1179-1190.
Artigo apresentado em 28/02/2018

Aprovado em 31/03/2018

Versão final apresentada em 02/04/2018 